\title{
Elementary algebra for Origami: The trisection problem revisited
}

\author{
Hiroyuki Shima \\ Department of Environmental Sciences \& Interdisciplinary Graduate School of Medicine and Engineering, University of Yamanashi, \\ 4-4-37, Takeda, Kofu, Yamanashi 400-8510, Japan
}

\section{Email address:}

hshima@yamanashi.ac.jp

\section{To cite this article:}

Hiroyuki Shima. Elementary Algebra for Origami: The Trisection Problem Revisited.American Journal of Applied Mathematics. Vol. 1, No. 4, 2013, pp. 39-43. doi: 10.11648/j.ajam.20130104.11

\begin{abstract}
This article presents an algebraic background in solving the angle trisection problem using origami-folding. Origami has been originally the art of paper folding, and recently aroused strong interest in a wide discipline of science and technology owing to its deep mathematical implication. Origami is also known to be an efficient tool for solving the trisection problem, one of the three famous problems of ancient Greek mathematics. Emphasis in this article is put on the way how the origami-based construction of the trisection corresponds to obtaining a solution for a cubic equation.
\end{abstract}

Keywords: Origami, Paper Folding, Angle Trisection, Construction Problem

\section{Introduction}

"Origami", a traditional game (or art) passed down through the ages, involves the creation of various shapes by folding a single sheet of paper. Variations in the created pieces are diverse and range from the imitation of animals, plants and tools, used in daily life, to abstract and complex three-dimensional structures. Beautiful works devised by implementing different techniques delight the eyes of beholders [1].

The origin of origami in Japan can be traced back to a record of the origami culture in a historical document from the 17 th century. Furthermore, it has also been known that an origami culture, independent from the one in Japan, existed in European countries, such as Germany and Spain, at least by the 19th century. The origami culture, which developed independently in individual countries and separate regions, has mutually amalgamated over a long period of time since the 20th century and as a result it is now attracting attention as a formative art that surpasses the realm of simple games.

A strong interest has emerged in the mathematics that lie deep within the techniques of origami, aside from the pleasure of "folding paper to create works of art", particularly since the beginning of the 21 st century [2]. Furthermore, proposals for application technologies, inspired by the process and also by the forms of origami created works, are starting to be made in a broad range of science and technology fields. Structural mechanics $[3,4,5]$, material chemistry [6,7], computational design [8] and physics of the early universe [9] can be cited as just a few such academic examples. It would be fair to say that such origami-inspired science and technology is still in its early stages of development and that the discovery of the usability of the knowledge of origami from a variety of perspectives is likely to continue in the future.

When considering the relevance of origami in the field of mathematics in particular, the most interesting topic would be a solution to the three famous problems of ancient Greek mathematics using origami drawings. The so called three famous problems of ancient Greek mathematics involve trisecting an angle, duplicating a cube and squaring a circle, which in any case are impossible to draw when using only a straight edge and a compass [10]. From the algebraic viewpoint the reason the two former problems cannot be solved lies in the fact that they require a solution to a cubic equation. In other words, these two problems both require drawing a segment with a length of the third root $\sqrt[3]{x}$ in relation to the segment with length $x$. A drawing method based on the use of a straight edge and compass alone, however, can only draw a segment of a line with a length that is a square root $\sqrt{x}$ (in other words it is possible to solve only up to a quadratic equation). On the other hand, origami-based drawing through the process of "folding paper" enables us to find a solution to a cubic equation and to trisect any arbitrary angle. This fact was demonstrated in 
a concise manner by H. Abe in 1980 [11], though already implied by M. Beloch in 1936 [12]. Since Abe's work was published in a document written in Japanese, it initially did not draw a lot of attention. But as soon as it was presented in English afterword, its importance was recognized instantly and it became known throughout the world [13]. (a)

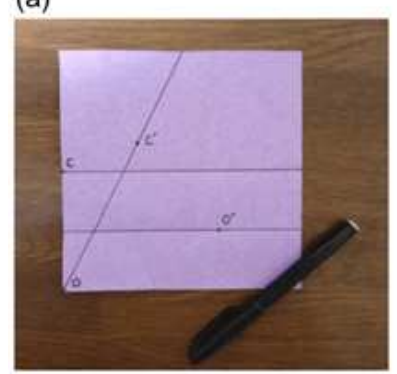

(c)

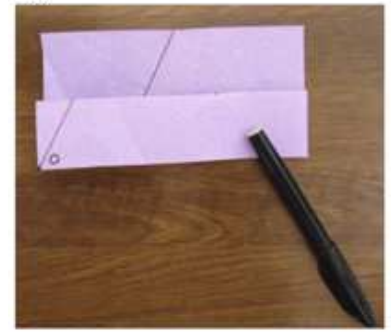

(b)

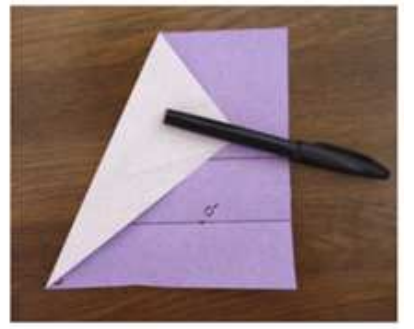

(d)

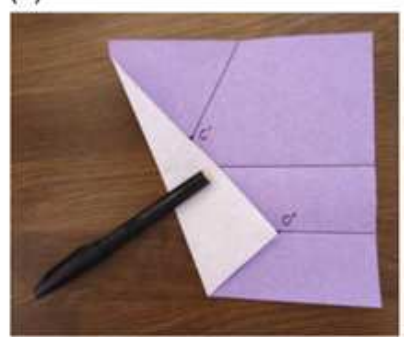

Figure 1. Appearance in process of trisection by Origami. The symbols and lines drawn in the paper correspond to those defined in Fig. 2.

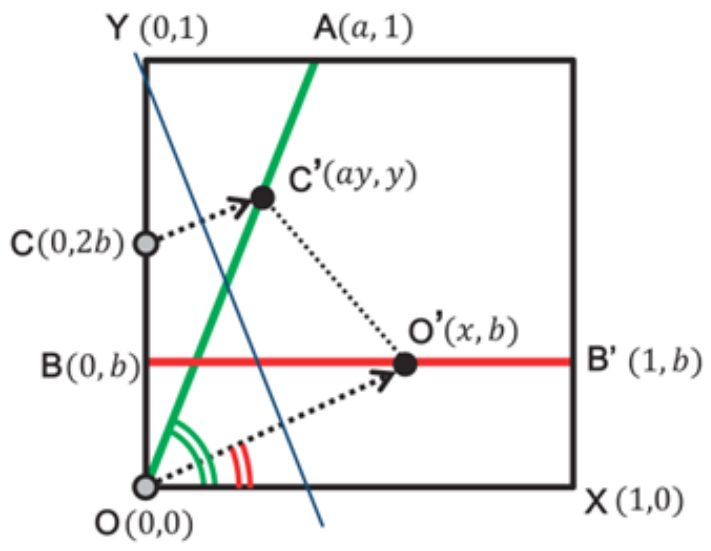

Figure2. Diagram of drawing the trisector (= the segment $O O^{\prime}$ ) of the given angle $X O A$.

This paper presents multiple algebraic ways to prove Abe's method for solving the trisection problem, as well as a concise explanation on how the method corresponds to obtaining a solution for a cubic equation. The mathematical derivation used are limited to an undergraduate level; therefore, it is content that would be suitable as teaching materials for elementary geometry intended for students in their freshman year at university. I would like to invite the readers to actually take a piece of paper and fold it to truly experience the advanced mathematics hidden beneath.

\section{Trisection Process}

Let's just start with, prepare a piece of palm-sized paper with square shape. For convenience, the one-side length of the square is supposed to be unit, and the origin $\mathrm{O}(0,0)$ is assigned to one vertex of the square. Fig.2 illustrates the definitions of variables and symbols used in the following discussion.

Here is the recipe of the trisection by origami:

1. Set the angle $\angle \mathrm{XOA}$ arbitrary.

2. Make a crease line along the slanted segment OA.

3. Make two more crease lines BB' and CC', which are both horizontal. Here, the point $\mathrm{C}$ " locates on the line XB'. The three horizontal segments, OX, BB', and CC", must be evenly spaced with each other.

4. Make another crease along a counterclockwise-slanted segment depicted in Fig. 2 (thin solid). By folding the paper along the crease, the points $\mathrm{O}$ and $\mathrm{C}$ are superimposed to $\mathrm{O}^{\prime}$ and C', respectively, each of which must be located on the lines BB' (colored in red) and OA (green).

5. Consequently, the obtained segment OO' is the trisector of the angle $\angle \mathrm{XOA}$ that was initially defined.

Why does it become possible to perform a trisection of an angle when origami is used? The key to solving the secret is in process 4 described above.

In process 4 , two points $\mathrm{O}$ and $\mathrm{C}$ were "simultaneously" relocated to the above segments $\mathrm{BB}$ ' and $\mathrm{OA}$ respectively. This is actually a process unique to origami and one that cannot be executed by Euclidean geometrical drawings that use a straight edge and a compass. In fact, the only procedure permitted in the drawing method of the latter is the following two:

- Connecting two points with a straight line.

- Drawing a circle with the center at a point.

Therefore, the process of superimposing two different points onto two straight lines "simultaneously" is equivalent to the introduction of a new axiom that is beyond the scope of Euclidean geometry [14,15]. A further remarkable fact is that, only by introducing the additional axiom in the Euclidean plain geometry, we are becoming able to draw geometrical objects that are beyond the ordinary drawing method based on straightedge and compass. Those who are interested in the issue are recommended to refer to the literature [2] for instance.

\section{Algebraic Expression}

To proceed algebraic argument, we pay attention to the tetragon $\mathrm{OO}^{\prime} \mathrm{C}^{\prime} \mathrm{C}$ presented in Fig. 2 and its geometric properties. By definition, it is a trapezoid having the two bases. See the panel (a) in Fig. 3, wherein the two parallel 
(a)

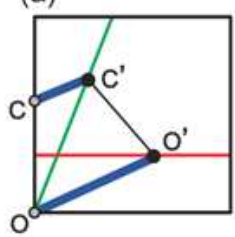

(b)

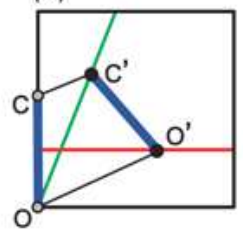

(c)

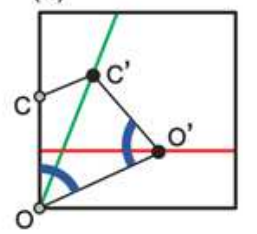

Figure3. Geometric feature of the trapezoid $O O^{\prime} C^{\prime} \mathrm{C}$. (a) Parallelism in the two bases $O O^{\prime} / / C C^{\prime}$, (b) Isometry in the two legs $O C=O^{\prime} C^{\prime}$; (c) Isogonality in the two base angles $\mathrm{COO}^{\prime}=\mathrm{C}^{\prime} \mathrm{O}^{\prime} \mathrm{O}$.

bases are highlighted by thick lines. Moreover, we observe that it is an isosceles trapezoid having the two legs with the same length [panel (b)] and the two base angles with the same measure [panel (c)].

The three properties of the trapezoid are expressed algebraically as follows:

1. The parallelism in the two bases, OO' $\| \mathrm{CC}^{\prime}$ :

$$
\frac{y-2 b}{a y}=\frac{b}{x} .
$$

2. The isometry in the two legs, $\mathrm{OC}=\mathrm{O}^{\prime} \mathrm{C}^{\prime}$ :

$$
(x-a y)^{2}+(y-b)^{2}=(2 b)^{2} .
$$

3. The isogonality in the two base angles, $\angle \mathrm{COO}^{\prime}=\angle$ C'O'O:

$$
\frac{x}{b}=\frac{\left(\frac{b}{x}\right)+\left(\frac{y-b}{x-a y}\right)}{1-\left(\frac{b}{x}\right) \cdot\left(\frac{y-b}{x-a y}\right)}
$$

The left and right sides in (3) equal to $\tan (\angle \mathrm{COO}$ ') and $\tan \left(\angle \mathrm{C}^{\prime} \mathrm{O}^{\prime} \mathrm{O}\right)$, respectively. To derive the right-side expression, we have used the additive theorem: $\tan (\alpha+$ $\beta)=(\tan \alpha+\tan \beta) /(1-\tan \alpha \tan \beta)$ with $\alpha=\angle$ OO'B $^{\prime}$ and $\beta=\angle \mathrm{C}^{\prime} \mathrm{O}$ 'B.

As a result, we can rephrase the trisection problem as the system of algebraic equations (1)-(3) with respect to $x$ and $y$, for given constants $a$ and $b$. We will see below that the system of three equations is reduced to one cubic equation with respect to $x$, which uniquely determines the way how to fold the squared paper "origami" to draw a trisector.

\section{The Cubic Equation}

To derive the key equation in terms of $x$, we eliminate $y$ from the system of three equations aforementioned. It follows from (1) that

$$
y=\frac{2 b x}{x-a b} .
$$

Substitute it to (2) to yield

$$
\left(x-\frac{2 a b x}{x-a b}\right)^{2}+\left(\frac{2 b x}{x-a b}-b\right)^{2}=4 b^{2}
$$

which can be simplified as

$$
(x-3 a b)\left(x^{3}-3 a b x^{2}-3 b^{2} x+a b^{3}\right)=0 .
$$

In a similar manner, we eliminate $y$ from (3) to derive

$$
-x\left(x^{3}-3 a b x^{2}-3 b^{2} x+a b^{3}\right)=0 .
$$

The two results, given by (6) and (7), indicate that our remained task is to solve the cubic equation $f(x)=0$ with the definition

$$
f(x)=x^{3}-3 a b x^{2}-3 b^{2} x+a b^{3} .
$$

In principle, the equation $f(x)=0$ gives us three roots of $x$. Among them, the only solution satisfying $0<x<1$ (and $0<y<1$ ) is required; otherwise, it is not relevant for solving the trisection problem, as readily understand from Fig. 2.

\section{Three Real Roots}

The famous Cardano's formula allows us to write the three roots of $f(x)=0$, designated by $x_{j}(j=0,1,2)$, as listed below:

$$
x_{j}=a b+\left(1+a^{2}\right)^{\frac{1}{2}} b\left[\omega_{j} \frac{(i+a)^{\frac{1}{3}}}{\left(1+a^{2}\right)^{\frac{1}{6}}}+\omega_{j}^{*} \frac{\left(1+a^{2}\right)^{\frac{1}{6}}}{(i+a)^{\frac{1}{3}}}\right] .
$$

Here, $\omega_{j}(j=0,1,2)$ describes the cubic root of one:

$$
\omega_{0}=1, \quad \omega_{1}=-\frac{1+i \sqrt{3}}{2}, \omega_{2}=\omega_{1}^{*},
$$

and the asterisk indicates to take complex conjugate. Despite the imaginary unit $i$ involved in (9), all the three roots are found to be real numbers. To make it clear, we set $(i+a)^{\frac{1}{3}}=r e^{i \varphi}$ to obtain

$$
\frac{(i+a)^{\frac{1}{3}}}{\left(1+a^{2}\right)^{\frac{1}{6}}}=\frac{r e^{i \varphi}}{r}=e^{i \varphi} .
$$

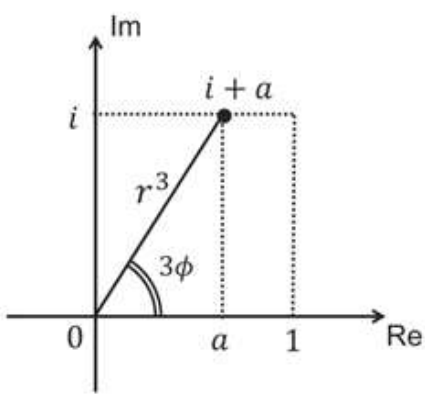

Figure4. Schematics of the polar-coordinate expression of the complex value: $i+a=r^{3} e^{3 i \varphi}$.

Fig.4 shows the geometric meanings of the polar coordinates $r$ and $\varphi$. Due to the condition of $0<a<1$, the angle $\varphi$ is restricted to the range of $0<\varphi<\pi / 6$. Then, the expression (9) is simplified as

$$
x_{0}=a b\left(1+2 \frac{\cos \varphi}{\cos 3 \varphi}\right), x_{1,2}=a b\left(1+2 \frac{\cos \left(\varphi \pm \frac{\pi}{3}\right)}{\cos \left[3\left(\varphi \pm \frac{\pi}{3}\right)\right]}\right)
$$

where $\varphi+\frac{\pi}{3}\left(\right.$ or $\left.\varphi-\frac{\pi}{3}\right)$ corresponds to $x_{1}\left(x_{2}\right)$. In the 
derivation, we used the relations

$$
\cos 3 \varphi=\frac{a}{\left(1+a^{2}\right)^{\frac{1}{2}}} \text { and } \cos 3 \varphi=-\cos \left[3\left(\varphi \pm \frac{\pi}{3}\right)\right]
$$

\section{Which Root Is Relevant?}

The expressions in (12) are further simplified by using the relation $\tan 3 \varphi=1 / a$ as well as the triple-angle formula for trigonometric functions. After manipulation, we obtain the results:

$$
x_{0}=\frac{b}{\tan \varphi}, x_{1}=\frac{-b}{\tan \left(\varphi+\frac{\pi}{3}\right)}, x_{2}=\frac{-b}{\tan \left(\varphi-\frac{\pi}{3}\right)} .
$$

Of these, only $x_{0}$ is the relevant solution, providing the way how to draw the trisector. In fact, it implies that $b / x_{0}=\tan \varphi$, or equivalently (see Fig. 2)

$$
\angle \mathrm{O}^{\prime} \mathrm{OX}=\varphi
$$

under the condition of

$$
\angle \mathrm{AOX}=3 \varphi .
$$

Eventually, we have secured the recipe for the trisection problem: Given the two constants $a$ and $b$, fix the angle $\varphi=\arg (i+a) / 3$ and then $x_{0}=b / \tan \varphi$. The point $\mathrm{O}$ ' $\left(x_{0}, b\right)$ tells us the trisector OO' that we want.

Note that the second solution, $x_{1}$, is irrelevant because it is always negative for every choices of $a$ and $b$ as long as they reside from 0 to 1 . The third one, $x_{2}$, is also irrelevant although it is positive; the irrelevance stems from that $x_{2}$ makes $y$ always negative for any choices of $a$ and $b$. In fact, substituting $x_{2}$ to (4) we find

$$
y=2 b\left[1+\frac{\tan \left(\varphi-\frac{\pi}{3}\right)}{\tan 3 \varphi}\right]^{-1}<0 \text { for } 0<\varphi<\frac{\pi}{6}
$$

\section{Concluding Remark}

It warrants notice that the cubic equation $f(x)=0$ we have considered is possible to be deduced in a simpler way, if we take into account the formula

$$
\tan 3 \varphi=\frac{3 \tan \varphi-\tan ^{3} \varphi}{1-3 \tan ^{2} \varphi}
$$

Let us go back to Fig. 2. The whole aim was to evaluate the appropriate value of $x$ that satisfy

$$
\tan \varphi=\frac{b}{x} \text { and } \tan 3 \varphi=\frac{1}{a} .
$$

If we substitute them into the formula (18), then we obtain

$$
\frac{1}{a}\left[1-3\left(\frac{b}{x}\right)^{2}\right]=3\left(\frac{b}{x}\right)-\left(\frac{b}{x}\right)^{3}
$$

which is equivalent to the cubic equation $f(x)=0$. Therefore, it is trivial that:
- The cubic equation $f(x)=0$ is an algebraic expression of the trisection problem, and

- The equation has at least one root $x$ that satisfies the desired relations of (19).

Hence, so to speak, the cubic equation $f(x)=0$ had been already solved in part before we've even started the calculation; what we have done are: i) the evaluation of other two (irrelevant) roots, and ii) the algebraic proof that no multiplicity exists in the way of paper folding [11] for obtaining the trisector.

In conclusion, I have provided a concise explanation on the origami-based trisection of an arbitrary angle. The method, originally suggested by H. Abe in 1980, enables us to draw a trisector of an arbitrary angle, thus being powerful than the ordinary Euclidean method based on straightedge and compass. I have demonstrated what kind of cubic equation describes the trisection problem and which root of it determines the way of paper folding for obtaining the trisector. I hope this expository article would give for readers a source of intellectual stimulus on the origami algebra.

\section{Acknowledgements}

The work was supported in part by Grant-in-Aid for Scientific Research from the Japan Society for the Promotion of Science (Grant No. 25390147) and JGC-S Scholarship Foundation. The author thanks my twin young daughters, Yuuka and Ayaka, who requested me to make toys by origami so repeatedly that have sparked the author's interest in the current issue.

\section{References}

[1] K. Kasahara: "Origami Omnibus: Paper-Folding for Everybody" (Japan Publication Inc., 1998)

[2] R. Geretschläger: "Geometric Origami" (Arbelos, UK, 2008).

[3] M. A. Dias, L. H. Dudte, L. Mahadevan and C. D. Santangelo: "Geometric Mechanics of Curved Crease Origami", Phys. Rev. Lett. 109 (2012) 114301.

[4] Z.Y. Wei,Z.V. Guo, L. Dudte, H.Y. Liang, and L. Mahadevan: "Geometric Mechanics of Periodic Pleated Origami", Phys. Rev. Lett. 110 (2013) 215501.

[5] M. Schenk and S. D. Guest: "Geometry of Miura-folded metamaterials", Proc. Natl. Acad. Sci. USA 110 (2013) 3276 .

[6] M. S. Strano: "Functional DNA Origami Devices", Science 338 (2012) 890.

[7] J. S. Siegel: "Carbon Origami”, Nature 486 (2012) 327.

[8] J. Hoffman: “The origami geometer", Nature 483 (2012) 274.

[9] N. Kaloper: "Origami world”, J. High Energ. Phys. 05 (2004) 061. 
[10] A. Jones, S. A. Morris and K. R. Pearson, “Abstract Algebra and Famous Impossibilities", (2ed., Springer-Verlag, 1994)

[11] H. Abe: "Possibility of trisection of arbitrary angle by paper folding" in SUGAKU Seminar (in Japanese) (Suken Publishing, Kyoto, 1980).

[12] M. P. Beloch: "Sulmetododelripiegamontodella carte per la risouzionedeiproblemigeometricic", Periodico di MathematicheSerie IV, 16 (1936) 104.
[13] T. Hull: "A note on impossible paper-folding", Am. Math. Month. 103 (1996) 242.

[14] H. Huzita: "Axiomatic development of origami geometry", in Proc. of the 1st Int'l Meeting of Origami Science and Technology (1989) pp.143-158.

[15] R. C. Alperin: "A mathematical theory of origami constructions and numbers", New York J. Math. 6 (2000) pp.119-133. 